\title{
Contained myocardial rupture: a variant linking complete and incomplete rupture
}

\section{T A Helmy, W J Nicholson, S Lick, B F Uretsky}

Heart 2005;91:e13 (http://www.heartjnl.com/cgi/content/full/91/2/e13). doi: 10.1136/hrt.2004.048082

\begin{abstract}
Myocardial rupture is an uncommon complication of myocardial infarction, often with devastating haemodynamic consequences. Although rupture is usually fatal, when patients do survive, the majority present with a pseudoaneurysm in which the rupture is sealed by a haematoma on the epicardial surface of the heart. Cases in which all myocardial layers are dissected except the epicardium or visceral pericardium have been included under this subheading. The authors describe such a case and suggest the pathological description of a "contained myocardial rupture". This link between complete and incomplete myocardial rupture may allow a more conservative management approach to be pursued.
\end{abstract}

M yocardial rupture is an uncommon complication of myocardial infarction, often with devastating haemodynamic consequences. After cardiogenic shock, it is the leading cause of in-hospital mortality after myocardial infarction. ${ }^{1}$ Although rupture is usually fatal, some patients may survive. Perdigao et $a l^{2}$ and Sutherland et $a l^{3}$ independently described four distinct morphological patterns of myocardial rupture (table $\mathrm{l}^{4}$ ). When patients do survive, the majority present with a type III or IV rupture. A type III rupture is described as one protected either by thrombus formation on the endocardial side of the orifice or by a pericardial symphysis on the epicardial surface. In a type IV rupture, the ventricular wall is typically thinned and dilated but the rupture does not extend through all layers of the myocardium. Both type III and type IV ruptures are often grouped together as pseudoaneurysms. Reports in which all myocardial layers are dissected except the epicardium or visceral pericardium have been included under the broad term "pseudoaneurysm". ${ }^{4}$ "The lack of direct communication between the left ventricular and the pericardial cavities is a common feature in ruptures roofed by haematoma and those roofed with visceral pericardium. While a rupture roofed by haematoma is a true pseudoaneurysm, we suggest that a rupture roofed by visceral pericardium be described as a "contained myocardial rupture" being a link between a complete and incomplete myocardial rupture.

\section{CASE REPORT}

A 47 year old man presented to the emergency department with chest discomfort and new ST segment depressions. Cardiac catheterisation was performed and showed a totally occluded circumflex lesion and a 90\% mid left anterior descending (LAD) artery lesion. Acute balloon angioplasty of the circumflex was performed. The patient developed recurrent angina and acute ST segment elevation in the precordial leads the following day. Repeat catheterisation with angioplasty and stenting of the mid LAD lesion were performed. Left ventriculography showed only inferior wall hypokinesis.

The patient continued to have symptoms of intermittent chest pain without ECG changes. An echocardiogram was performed for concern of a possible ventricular free wall rupture. A moderate pericardial effusion and a wall motion abnormality in the posterior-inferior-lateral wall of the left ventricle were seen. A narrow neck of communication between the left ventricular chamber and the pericardial space could not be clearly identified (fig lA).

Repeat catheterisation showed the LAD and circumflex coronary arteries to be widely patent. Ventriculography showed a large bulge beyond the normal contour of the inferolateral wall, directly below the mitral annulus (fig 1B). There was no evidence of additional dye extravasation into the pericardial space.

Owing to the presence of the pericardial effusion in the presence of a possible pseudoaneurysm, surgery was performed. On direct surgical inspection a firm mass of about $2.5 \mathrm{~cm}$ was found on the inferolateral wall of the left ventricle with circumferential thinning of the surrounding myocardium (fig 2). The mass consisted of a contained myocardial rupture covered by a roof of intact visceral pericardium. The endoventricular wall was repaired with a pericardial patch with full thickness sutures anchored with felt on the outside of the ventricle with subsequent approximation of the epicardium.

\section{DISCUSSION}

This case illustrates the interesting entity of contained myocardial rupture. Both an echocardiogram and a ventriculogram were unable to distinguish whether the defect was a pseudoaneurysm or a true aneurysm. Surgery showed a type III rupture, with complete disruption of an area of myocardium but with an intact, thinned, and fibrotic visceral pericardium.

This case is an example of the diagnostic dilemma sometimes encountered when there is near penetration into the pericardial space. The natural history of this variant of myocardial rupture is unclear, since most published reports

Table 1 Four morphological patterns of myocardial rupture $^{4}$

\begin{tabular}{ll} 
Type & Description \\
\hline I & $\begin{array}{l}\text { Nearly direct trajectory of rupture through the entire myocardium } \\
\text { with little dissection or bloody infiltration of the myocardium }\end{array}$ \\
II $\quad \begin{array}{l}\text { Multicanalicular trajectory with extensive myocardial dissection } \\
\text { and bloody infiltration of the myocardium }\end{array}$ \\
III $\quad \begin{array}{l}\text { Orifice of rupture on the external surface of the myocardium } \\
\text { protected either by thrombus or pericardial symphysis } \\
\text { Incomplete rupture; the trajectory does not extend through all } \\
\text { layers of the myocardium and is therefore not transparietal }\end{array}$ \\
IV
\end{tabular}



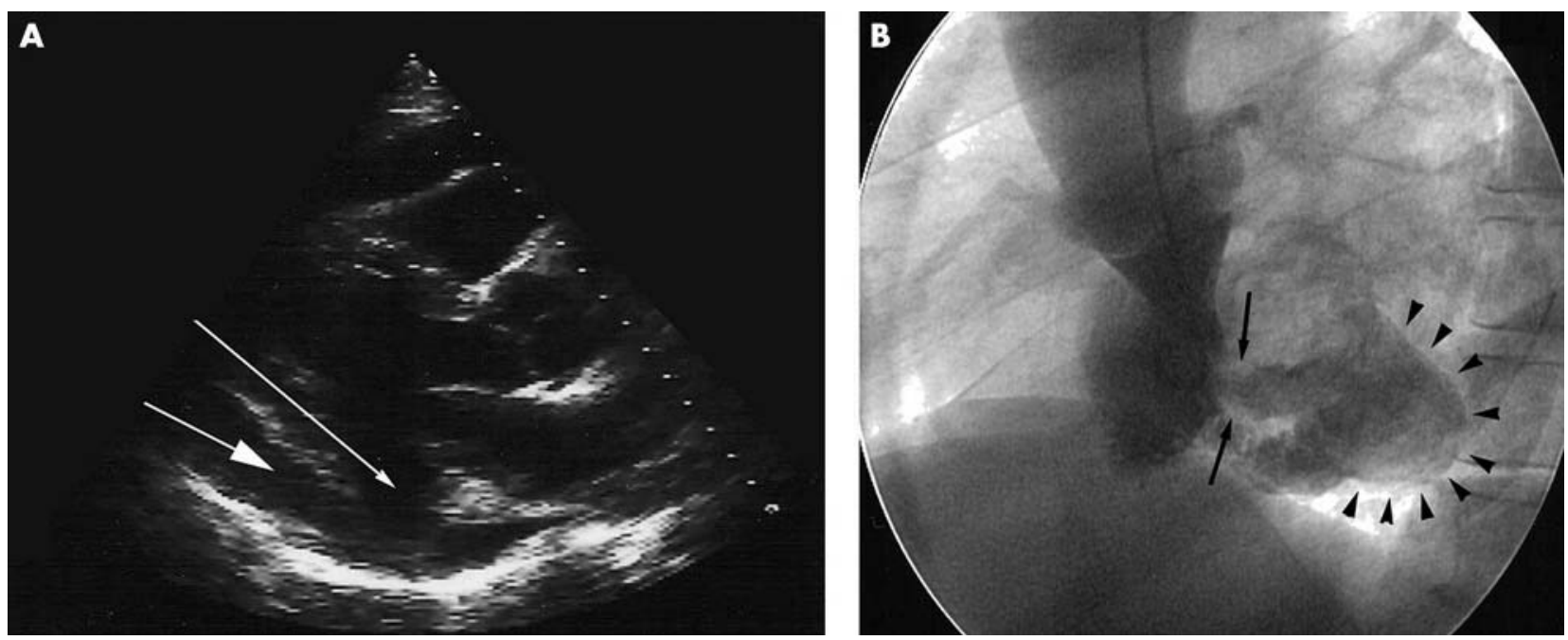

Figure 1 (A) Echocardiogram in the parasternal long axis view showing a defect in the posterior-inferior left ventricular wall (small arrow) and posterior pericardial effusion (large arrow). (B) Ventriculogram in the left anterior oblique view showing a bulge in the posterolateral wall of the left ventricle (arrowheads) connected to the ventricular chamber by a narrow well defined neck (arrows).

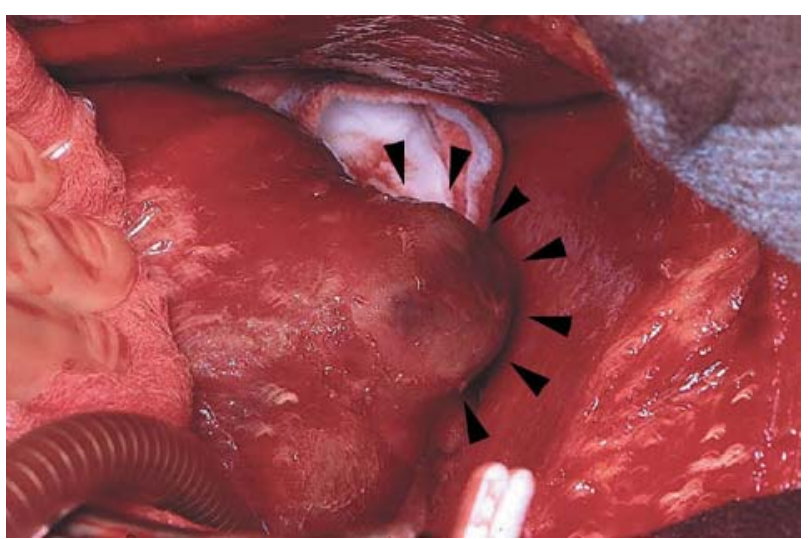

Figure 2 Intraoperative photograph of the firm mass of about $2.5 \mathrm{~cm}$ found on the inferolateral wall of the left ventricle with the intact visceral pericardium cover.

do not categorise contained rupture as a separate entity. Whether the absence of communication between the left ventricular and pericardial cavities provides more stability to the ventricular wall and protects it from subsequent frank rupture is unknown. This stability afforded by the pericardial roof may provide the patient with the additional time needed for the correct diagnosis to be made and acted upon.

Pseudoaneurysms in general carry a high incidence of rupture ranging between $30-45 \%$, leading to fatal tamponade. ${ }^{6}$ In contrast to the conservative treatment typically pursued in the presence of a true aneurysm, most authors feel that urgent surgery is the treatment of choice for pseudoaneurysms. However, in a series reported by Figueras et al, selected patients with left ventricular free wall rupture after infarction were acutely managed with fluids, inotropic support, and therapeutic pericardiocentesis. Patients were then followed up clinically without surgical intervention. Thirteen of 19 patients survived for an average of over four years. ${ }^{1}$ The possibility that some of these patients may have had a contained myocardial rupture was not pursued. It is possible that the presence of an epicardial roof may confer adequate stability to offer some protection against free bleeding into the pericardial space and the development of cardiac tamponade. The distinction between complete and contained myocardial rupture may be clinically valuable if a prognostic distinction can be seen. Improving diagnostic imaging modalities such as magnetic resonance imaging and transoesophageal echocardiography combined with awareness of this pathological distinction may further define the clinical course of patients with contained myocardial ruptures.

\section{Authors' affiliations}

T A Helmy, W J Nicholson, Emory University, Atlanta, Georgia, USA S Lick, B F Uretsky, University of Texas, Galveston, Texas, USA

Correspondence to: Dr Tarek A Helmy, Division of Cardiology, Emory University, 1639 Pierce Drive, Atlanta, Georgia 30322, USA; thelmy@ emory.edu

Accepted 11 August 2004

\section{REFERENCES}

1 Figueras J, Cortadellas J, Evangelista A, et al. Medical management of selected patients with left ventricular free wall rupture during acute myocardial infarction. J Am Coll Cardiol 1997;29:512-8.

2 Perdigao C, Andrade A, Ribiero C. [Cardiac rupture in acute myocardial infarction: various clinico-anatomical types in 42 recent cases observed over a period of 30 months]. Arch Mal Coeur Vaiss 1987;80:336-44.

3 Sutherland F, Guell F, Pathi V, et al. Postinfarction ventricular free wall rupture: strategies for diagnosis and treatment. Ann Thorac Surg 1996;61:1281-5.

4 Nathaniel C, Lane S, Palma R, et al. Pseudoaneurysm causing partial obliteration of the left atrium: case report and review. Cathet Cardiovasc Diagn 1996;38:83-6.

5 Lopez-Sendon J, Gonzalez A, De Sa E, et al. Diagnosis of subacute ventricular wall rupture after acute myocardial infarction: sensitivity and specificity of clinical, hemodynamic and echocardiographic criteria. J Am Coll Cardiol 1992; 19:1145-53.

6 Frances C, Romero A, Grady D. Left ventricular pseudoaneurysm. J Am Coll Cardiol 1998;32:557-61. 